\title{
Trending of SNPP ephemeris and its implications on VIIRS geometric performance
}

\author{
Guoqing (Gary) Lin*,a,b, Robert E. Wolfe ${ }^{\mathrm{b}}$, James C. Tilton ${ }^{\mathrm{c}}$ \\ a'Science Systems and Applications, Inc., 10210 Greenbelt Road, Lanham, MD, USA 20706; \\ bNASA Goddard Space Flight Center, Code 619, 8800 Greenbelt Rd, Greenbelt, MD, USA 20771; \\ 'NASA Goddard Space Flight Center, Code 606, 8800 Greenbelt Rd, Greenbelt, MD, USA 20771
}

\begin{abstract}
This paper describes trends in the Suomi National Polar-orbiting Partnership (SNPP) spacecraft ephemeris data over the four and half years of on-orbit operations. It then discusses the implications of these trends on the geometric performance of the Visible Infrared Imaging Radiometer Suite (VIIRS), one of the instruments onboard SNPP.

The SNPP ephemeris data includes time stamped spacecraft positions and velocities that are used to calculate the spacecraft altitude and sub-satellite locations. Through drag make-up maneuvers (DMUs) the orbital mean altitude (spacecraft altitude averaged over an orbit) has been maintained at $838.8 \mathrm{~km}$ to within $+/-0.2 \mathrm{~km}$ and the orbital period at 101.5 minutes to within $+/-0.2$ seconds. The corresponding orbital mean velocity in the terrestrial frame of reference has been maintained at $7524 \mathrm{~m} / \mathrm{s}$ to within $+/-0.5 \mathrm{~m} / \mathrm{s}$. Within an orbit, the altitude varies from $828 \mathrm{~km}$ near $15^{\circ} \mathrm{N}$ to $856 \mathrm{~km}$ near the South Pole. Inclination adjust maneuvers (IAMs) have maintained the orbit inclination angle at $98.67^{\circ}$ to with $+/-0.07^{\circ}$ and the sun-synchronous local time at ascending node (LTAN) at 13:28 to within $+/-5$ minutes. Besides these trends, it is interesting to observe that the orbit's elliptic shape has its major axis linking the perigee and apogee shorter than the line linking the ascending node and the descending node. This effect is caused by the Earth's oblate spheroid shape and deviates from a Keplerian orbit theory in which the two orbiting bodies are point masses.

VIIRS has 5 imagery resolution bands, 16 moderate resolution bands and a day-night band, with 32, 16 and 16 detectors, respectively, aligned in the spacecraft flight (aka. track) direction. For each band's sample within a scan, the detectors sample the Earth's surface simultaneously in the track direction in the Earth Centered Inertial frame of reference. The distance between the center of the area sensed by the trailing detectors of one scan and the leading detectors of the next includes a component caused by earth rotation. This earth rotation component is relatively small $(\sim 70 \mathrm{~m} / \mathrm{s})$ for an orbit like SNPP, but must be taken into account in the design of low-Earth orbit scanning sensors similar to VIIRS to ensure contiguous coverage at nadir.
\end{abstract}

Keywords: Suomi NPP, VIIRS, trends, ephemeris, altitude, inclination angle, ground speed, scan-to-scan overlap.

\section{INTRODUCTION}

The NASA/NOAA Visible Infrared Imaging Radiometer Suite (VIIRS) instrument onboard the Suomi National Polarorbiting Partnership (SNPP) satellite was launched on 28 October 2011. The instrument has performed well on-orbit ${ }^{[1,2,3]}$. Copies of VIIRS are planned to be on the four NOAA Joint Polar Satellite System (JPSS) satellites ${ }^{[3,4]}$. The JPSS satellites are planned to be in the same afternoon orbit as the SNPP but different phasing to increase near-nadir coverage when more than one VIIRS is operating. Since orbit parameters are used in the design of the SNPP and JPSS instruments, it is important to understand trends and variations of the parameters, and the possible impacts on the products from these instruments.

*gary.lin@nasa.gov; glin@ssaihq.com; phone 301-614-5451; fax 301-614-5269; www.ssai.org 
The VIIRS instrument onboard SNPP has 5 imagery resolution bands (I-bands), 16 moderate resolution bands (M-bands) and a day-night band (DNB). They respectively have 32, 16 and 16 detectors aligned with the spacecraft motion in the track direction. The nominal design of these bands is to have the field of regard (FOR) of $12 \mathrm{~km}$ on the ground in the track direction at nadir for the I-bands and M-bands and throughout a scan for the DNB. However, the actual track direction FOR varies depending on the actual fabricated detector size, VIIRS system effective focal length (EFL), and altitude of the carrying satellite. The actual VIIRS scan period $T$ and the SNPP sub-satellite point ground speed in the VIIRS track direction dictate how far the distance a VIIRS ground point travels from one scan to the next, $D_{\text {s2s }}$. Subsequently, a VIIRS scan-to-scan overlap occurs if an actual FOR is greater than $D_{s 2 s}$ while an underlap occurs if an FOR is narrower than $D_{s 2 s}$.

This paper first presents the trending results of the SNPP positions and speed in Section 2. Section 3 examines the orbit shape and variations of position and speed. Then the effects of orbit geometry on VIIRS coverage on the ground are examined in Section 4. Discussions and concluding remarks are then presented in Section 5.

\section{Trending of SNPP ephemeris data}

The SNPP ephemeris data is part of the spacecraft diary that includes attitude data and other telemetry data. The data is acquired on the spacecraft once a second and downlinked to the ground in a separate diary product, and then interleaved on the ground into each instruments' product for geolocation retrievals. The ephemeris data has the SNPP satellite time stamped position vectors and velocity vectors expressed in the Earth centered Earth fixed frame of reference. This reference frame is also called Earth centered rotating (ECR) frame, or terrestrial reference frame. With their time reference, the position and velocity vectors in ECR are transformed to their corresponding vectors in the Earth centered inertial (ECI) frame of reference, for which the orbit is a closed form of ellipse forming a plane within which the Earth rotates (see Section 3 below).

The spacecraft diary data for almost the entire mission has been used to calculate sub-satellite point (SSP) latitudes, longitudes, altitudes from the time-tagged satellite position and velocity vectors. Various parameters (see Fig. 1) were calculated from these time-tagged datasets for each orbit: local time at ascending node (LTAN); inclination angle; mean, minimum and maximum (not shown) altitude; mean, minimum (not shown) and maximum speed; period; and for every 16 days, the backward differences (BWD) of the SSP locations at the equator. As seen in Fig. 1(a), over more than four years the sun-synchronous LTAN initially started at 13:25, swung earlier to 13:23, swung later to 13:33 and then back earlier to 13:26. Fig. 1(b) shows that initially the inclination increased from about 98.65 degrees to about 98.75 degrees, almost monotonically. Before the third anniversary in the autumn of 2014, a campaign of four inclination adjustment maneuvers (IAMs) were conducted that brought the inclination down to about 98.63 degrees. As a consequence, the LTAN swung downward. But the inclination angle started to increase again and so in the autumn of 2015, another IAM (IAM\#5) was performed. The LTAN swung downward between IAM\#4 and IAM\#5 and continued to swing down after IAM\#5. Another IAM, IAM\#6, is planned to be performed in September 2016. The LTAN is expected to continue to swing down after IAM\#6. In the future, IAMs are expected to be performed about once a year to keep the LTAN close to 13:25 and inclination angle close to 98.67 degrees.

Besides the IAMs, another form of station-keeping operation that is regularly performed are the drag make-up maneuvers (DMUs). For SNPP, each DMU raises the orbit about $100 \mathrm{~m}$, keeping the orbital mean altitude to be about $838.8 \mathrm{~km}$, Fig. 1(c). Occasionally, debris avoidance maneuvers (also called risk management maneuvers or collision avoidance maneuvers) are performed. They are similar to DMUs in that they raise the orbit height. The purpose of the DMUs is to help keep the sun-synchronous ground track repeatable every 16 days and the orbital period near constant at 101.5 minutes, Fig. 1(e).

As a consequence of the DMU and IAM station-keeping operations, the orbit is kept "frozen", in that the orbit altitude, speed and LTAN are kept nearly constant. The orbital mean altitude has been maintained at $838.8 \mathrm{~km}$ to within $+/-0.2$ $\mathrm{km}$ peak-to-valley and the orbital mean speed (in the terrestrial frame of reference) is $7524 \mathrm{~m} / \mathrm{s}$ to within $+/-0.5 \mathrm{~m} / \mathrm{s}$ peakto-valley, Fig. 1(c) and (d). The minimum altitude and maximum speed are also kept near constant, at $828 \mathrm{~km}$ and 7538 $\mathrm{m} / \mathrm{s}$, respectively. The behaviors of maximum altitude and minimum speed are about the same. They are not shown here since they are not as much of a concern as the minimum altitude and maximum speed are, as will be shown in Section 4 . 
(a)

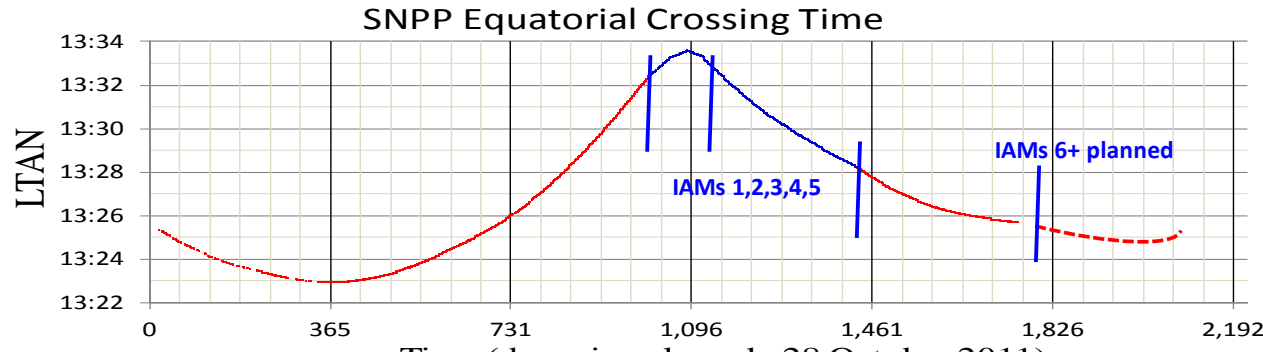

Time (days since launch, 28 October 2011)

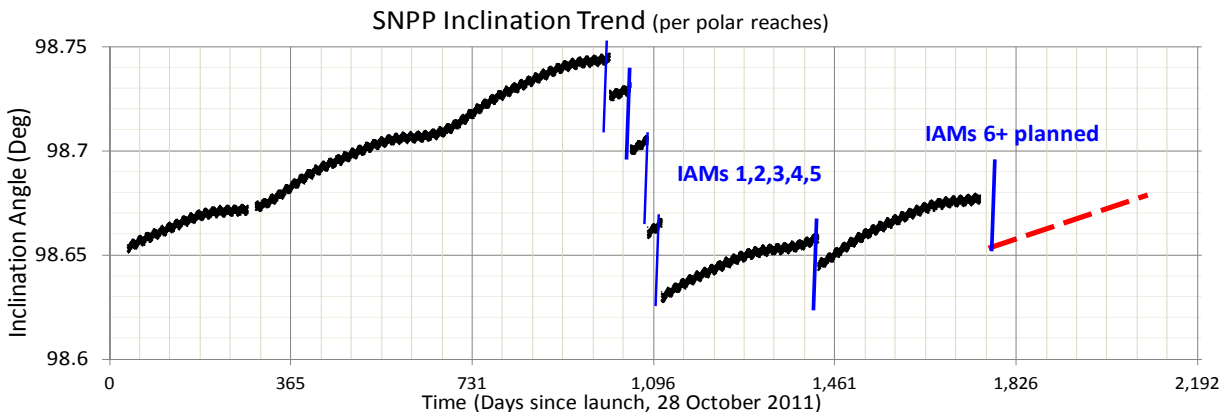

(b)

(c)

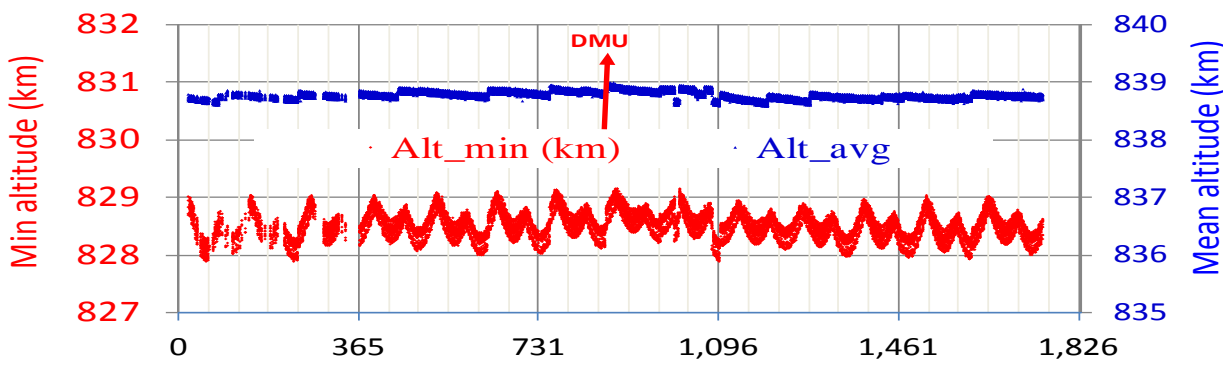

(d)
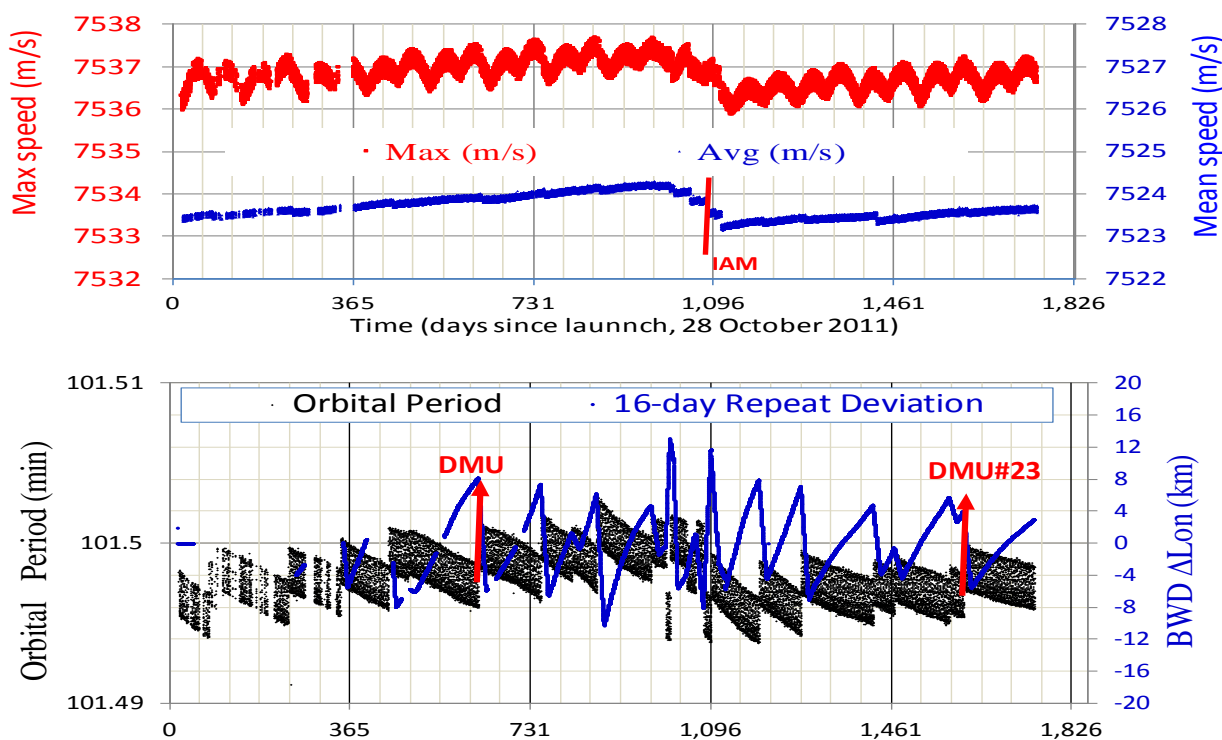

(e)

Time (Days since Launch on Oct 28, 2011)

Fig. 1. Trending of SNP ephemeris and derived parameters. (a) LTAN; (b) inclination angle; (c) mean and minimum altitude; (d) mean and maximum orbital speed in ECR; (e) orbital period and 16-day repeat ground track deviation. 


\section{Examination of parameters in one SNPP orbit}

Through the IAM and DMU station-keeping operations, the orbital minimum, mean, maximum speed and altitude are kept nearly constant, as shown in Fig.1 (c) and (d). However, the variations of these variables along an orbit path are relatively large. Data in the first full orbit on 30 October 2014 is examined in this section. Fig. 2(a) shows this orbit with its sub-satellite point (SSP) (or nadir) ground track. Fig. 2(b) shows the variations of the orbit (radial) distance from the Earth center and the speed in the ECR reframe frame plotted against the SSP latitude which varies from $81.3^{\circ} \mathrm{S}$ to $81.3^{\circ}$ N. Note that in Fig. 2(b) and (c), the vertical grid lines are spaced at every 203 seconds of time travelled in approximately $1350 \mathrm{~km}$ intervals along the ground track, but are labeled with the corresponding SSP latitude. This means that the amount of change in latitude varies between each pair of grid lines. In Fig. 2(b), the orbit radial distance varies by $+/-0.1 \%$, first decreasing about $10 \mathrm{~km}$ from the ascending node to near the North Pole, then back to about the same distance near the descending node and then increasing by about $6 \mathrm{~km}$ near the South Pole. In the same figure, the speed varies by $+/-0.3 \%$, decreasing by about $20 \mathrm{~m} / \mathrm{s}$ from the ascending node to the near North Pole, increasing back to about the same magnitude near the descending node and then decreasing by a larger amount to about $40 \mathrm{~m} / \mathrm{s}$ to near the South Pole.

(a)

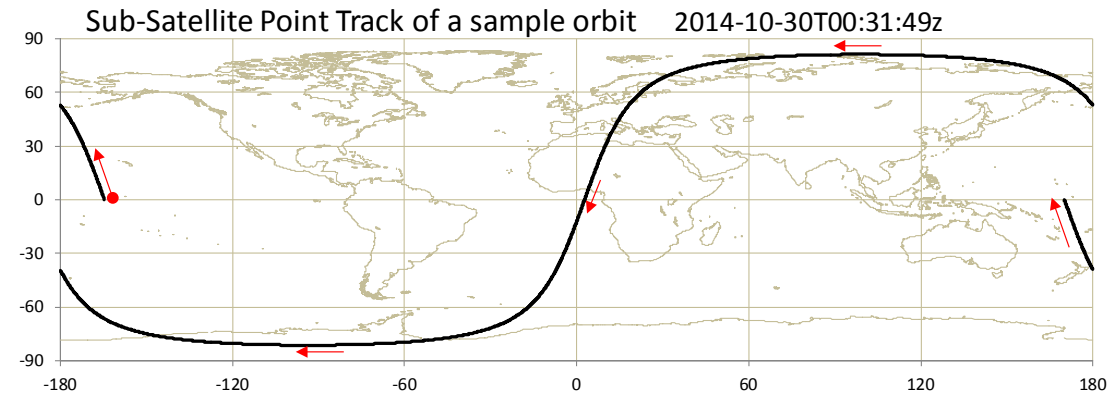

SNPP actual orbit position and speed in ECR

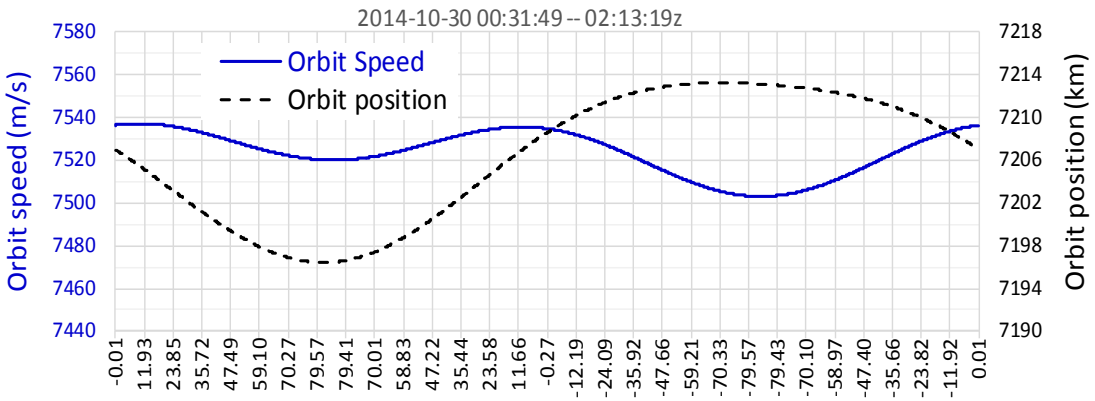

(b)

Latitude (deg) along the ground track

SNPP actual altitude \& ground speed in ECR

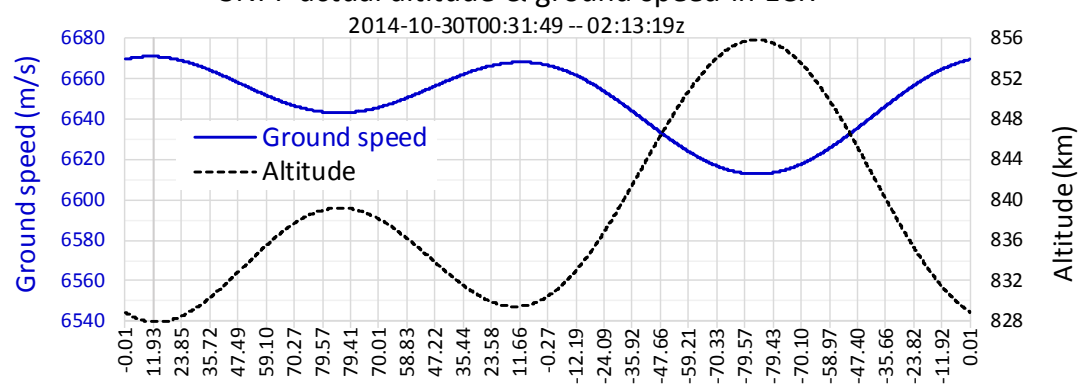

(c)

Latitude (deg) along the ground track

Fig. 2. A sample of a single SNPP orbit from ascending node to the next ascending node on 30 October 2014. (a) sub-satellite point (SSP) ground track with arrows showing the flight direction; (b) variations of satellite speed (in ECR) and distance from the Earth center (position); (c) variations of SSP speed (in ECR) and spacecraft altitude. See text for more information on the spacing between vertical grid lines. 
Relative to the Earth's surface (aka. the ground), the variations are larger, Fig. 2(c). The altitude varies by $+/-1.7 \%$, increasing from about $828 \mathrm{~km}$ near the ascending node to about $840 \mathrm{~km}$ near the North Pole, deceasing back to about 828 $\mathrm{km}$ near the descending node, and then increasing to $856 \mathrm{~km}$ near the North Pole. The SSP (ground) speed varies by +/$0.5 \%$, from 6670 to 6640 to 6670 to $6610 \mathrm{~m} / \mathrm{s}$, respectively, from the ascending node to the near North Pole to the descending node to the near South Pole.

The variations of spacecraft position (orbit radial distance), altitude and speed are relatively small and may be viewed more easily by removing the bulk of the magnitude and plotting in polar coordinates, Fig. 3 . The polar angle is the integrated orbit angle starting from the ascending node in the frame of reference. In ECI coordinates the orbit is in a plane so this orbit angle varies from $0^{\circ}$ to $360^{\circ}$, Fig. 4. However, in ECR coordinates, the path of an orbit is a spiral in three dimensions. Because the angular component of the Earth's rotation during the orbit is about $25^{\circ}$, the total angle is larger and so the range is from $0^{\circ}$ to $\sim 364.25^{\circ}$. This explains the small mismatch in the position between the beginning and end of the orbit (see inset in left figure).

In ECR coordinates, the point closest to the Earth surface is not at the ascending node nor at the North Pole, but somewhere north of the ascending node, where the fastest satellite speed is located. Because the radial distance near the North Pole is lower than that near the ascending node, the satellite speed should be faster, but instead it is slower which is a deviation from the classic Keplerian orbit theory ${ }^{[5]}$. For the point near the South Pole, the distance is larger than near the ascending node and the satellite speed is slower, obeying the classic Keplerian orbit theory. However, of course, it is not really appropriate to compare these parameters to a Keplerian orbit because the Earth's oblate shape causes the orbit to be nonKeplerian. Near the South Pole, where the spacecraft altitude is highest and the spacecraft speed is lowest, the SSP velocity is the slowest, as expected.
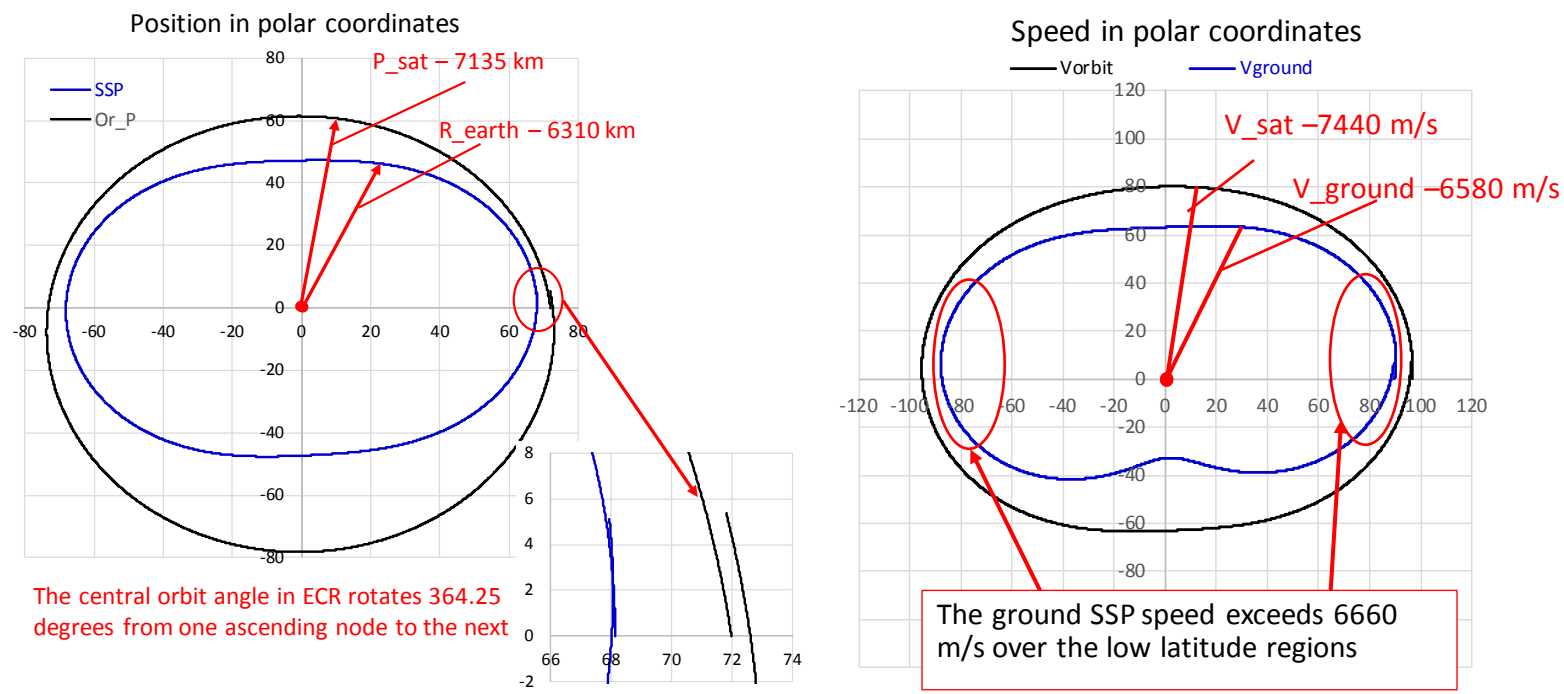

Fig. 3. Parameters for a sample SNPP orbit in ECR expressed in polar coordinates (after removing the bulk of the magnitude) as a function of the central orbit angle. (left) radial distance from the Earth center to the satellite position and SSP; (right) satellite speed and SSP (gound) speed.

In ECI coordinates, plots are made similar to Fig. 3, except that different subtracted values are used and plots of the mean position and speed are included to show subtle variations, see Fig. 4. We can see that the position is similar to ECR coordinates with a small difference due to the orbit central angle difference in the two reference frames. It is interesting to observe that the center of the orbit is located about $8.4 \mathrm{~km}$ south of the Earth's center. It is also interesting to observe that the major axis linking the perigee and the apoge ${ }^{[6]}$ is shorter than the line linking the ascending nodal point and the descending nodal point. This, again, deviates from the classic Keplerian orbit theory. However, when we look at the orbit speed distribution, the classic orbit theory nearly holds, where the speed is the fastest at the perigee and the slowest at apogee. 

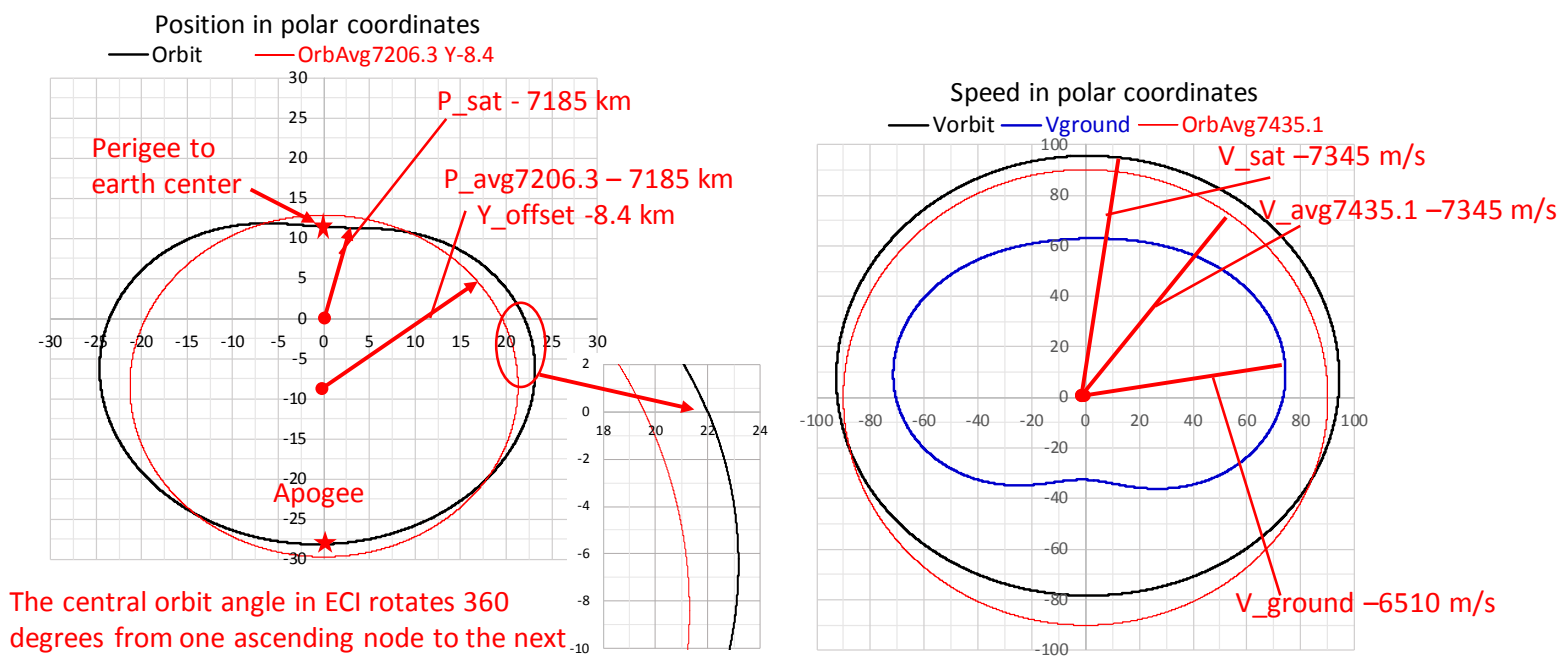

Fig. 4. A sample SNPP orbit parameters in the ECI frame expressed in polar coordinates (after removing the bulk of the magnitude) as a function of the central orbit angle. (left) satellite orbit position from the Earth's center and mean position shifted $8.4 \mathrm{~km}$ to the south of the Earth's center; (right) satellite orbit speed, mean orbit speed and ground speed.

For the ground speed in the ECI frame, the distribution is a little more constant than that in the ECR frame and the magnitude is smaller. The difference is due to earth rotation and the inclination angle. As we will see below, this difference plays a significant role in the design of instrument like VIIRS.

\section{Effects of orbit geometry on VIIRS ground coverage}

The VIIRS instrument onboard the SNPP satellite was designed to cover the Earth contiguously along the ground track of the SNPP orbit. Many factors must be included in the design of a scanning instrument like VIIRS to ensure that there is no gap between the scans, particularly at nadir during maximum SSP speed. The VIIRS detector arrays for all bands are aligned in the track direction. The rotating telescope assembly (RTA) rotates in the cross-track scan direction, forming a scan plane. The processes of design, fabrication, testing, integration into the spacecraft, and post-launch on-orbit calibration and validation are to ensure that the scan plane is to be perpendicular to the spacecraft track direction (although there is a very small rotation ("clocking") of the detector arrays about the optical axis to help with track direction bandto-band registration). The track direction is in the ECI frame of reference, since all detectors in one array register on the ground simultaneously, as shown in Figs. 5 and 6. Due to the orbit inclination angle of $98.67^{\circ}$ (for this orbit), the SSP ground track reaches the northern most latitude of $81.33^{\circ}$, see Fig. 5. At this point in the ECI frame, the spacecraft SSP travels west at $6573 \mathrm{~m} / \mathrm{s}$, and the Earth rotates at $70 \mathrm{~m} / \mathrm{s}$ eastward. For an observer on the ground (a fixed location in ECR), the Earth's rotation in ECI makes the satellite (and SSP) appear to be moving faster. So in the ECR frame the SSP velocity increases to $7643 \mathrm{~m} / \mathrm{s}$ by adding $-\mathrm{V}_{\text {earth }}$ to $\mathrm{V}_{\mathrm{ECI}}$.

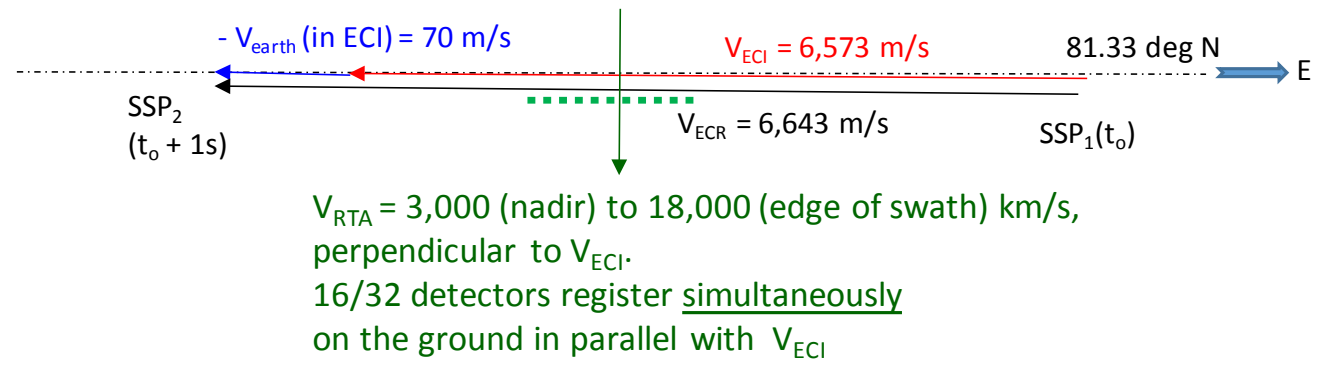

Fig. 5. Relationship of SSP and earth surface speed in ECI and ECR at the northern most part of the orbit. 
At the ascending node (Fig. 6), the SSP velocity is $6584 \mathrm{~m} / \mathrm{s}$ in the ECI frame and is "inclined" $98.67^{\circ}$. The earth rotates at a speed of $464 \mathrm{~m} / \mathrm{s}$ eastward in the ECI frame. For an observer on the ground, this means the satellite is moving faster westward than the SSP velocity of $6584 \mathrm{~m} / \mathrm{s}$ in the ECI frame at the inclined angle of $98.67^{\circ}$. The combined effect is that the SSP track travels at a speed of $6670 \mathrm{~m} / \mathrm{s}(86 \mathrm{~m} / \mathrm{s}$ larger) in the ECR frame. However, we are concerned about the distance between two scan centers on the Earth's surface. Since the scan plane is perpendicular to $V_{E C I}$, not $V_{E C R}$, the component of the Earth's surface velocity in the direction of $\mathrm{V}_{\mathrm{ECI}}$ must be added to $\mathrm{V}_{\mathrm{ECI}}$ to compute the distance between two scan centers. At the ascending node, this component is $70 \mathrm{~m} / \mathrm{s}$ so the corresponding velocity for computing the ground distance from one scan-center to the next $\left(\mathrm{V}_{\mathrm{s} 2 \mathrm{~s}}\right)$ is $6654 \mathrm{~m} / \mathrm{s}$.

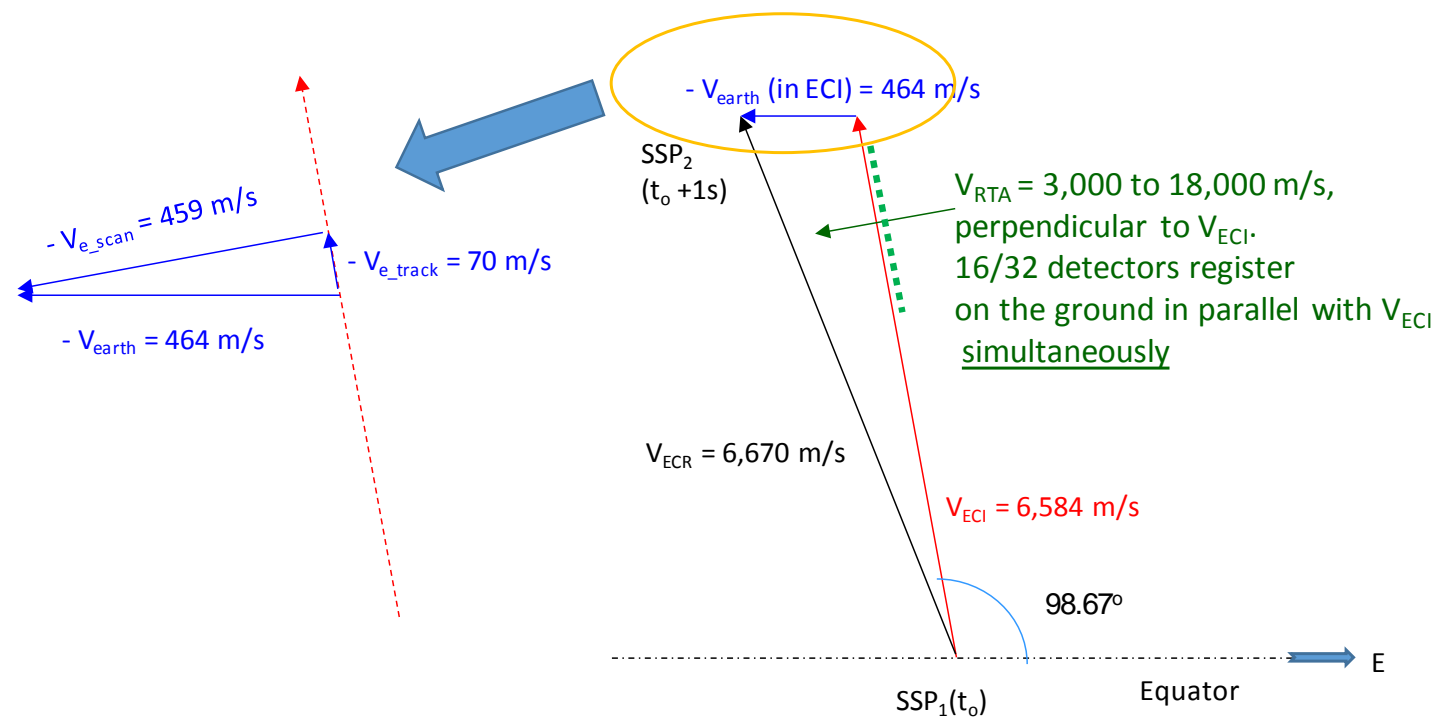

Fig. 6. Relationship of SSP and earth surface speed in ECI and ECR at the ascending node.

At the descending node and the southernmost reach of the SNPP ground track, the same calculation shows that the earth rotation contributes $70 \mathrm{~m} / \mathrm{s}$ to $V_{\mathrm{s} 2 \mathrm{~s}}$. Further, the earth rotation contributes $70 \mathrm{~m} / \mathrm{s}$ to $\mathrm{V}_{\mathrm{s} 2 \mathrm{~s}}$ everywhere in the orbit, as shown in Fig. 7. As we can see, $V_{\mathrm{s} 2 \mathrm{~s}}$ is the same as $\mathrm{V}_{\mathrm{ECR}}$ at the southernmost and the northernmost reaches, but smaller everywhere else. The largest difference is at the ascending node and descending node, at $0.24 \%$.

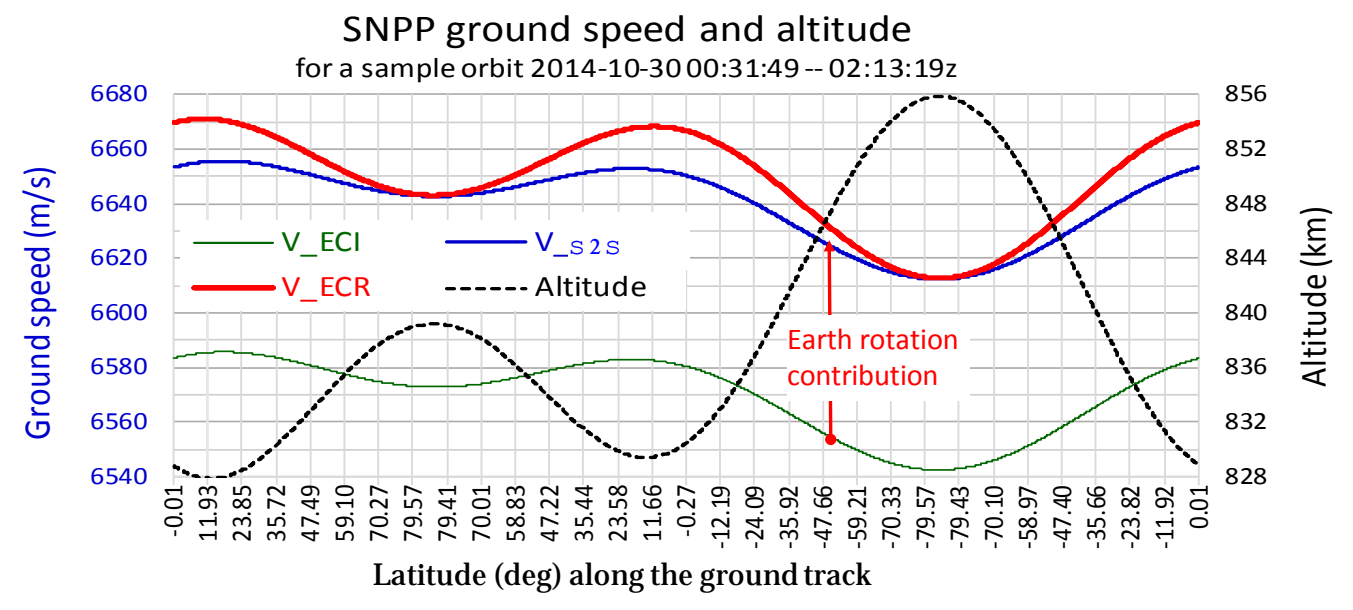

Fig. 7. Contribution of earth rotation to $V_{\mathrm{s} 2 \mathrm{~s}}$. $\mathrm{V}_{\mathrm{ECR}}$ is larger than $\mathrm{V}_{\mathrm{s} 2 \mathrm{~s}}$ except near poles.

From Figs. 5 and 6, the equation that gives the difference between $V_{\mathrm{ECI}}$ and $\mathrm{V}_{\mathrm{s} 2 \mathrm{~s}}$ at any point in an orbit is:

$$
\mathrm{V}_{\mathrm{s} 2 \mathrm{~s}}=\mathrm{V}_{\mathrm{ECI}}-\mathrm{V}_{\text {eartho }} \cos (i)
$$

where $V_{\text {eartho }}$ is the Earth surface's rotational speed in ECI coordinates at the equator and $i$ is the inclination. 
Let $T$ be the VIIRS scan period, $D_{\text {s2s }}$ be the distance that the center of the scan advances one scan to the next. We have

$$
\mathrm{D}_{\mathrm{s} 2 \mathrm{~s}}=T \mathrm{~V}_{\mathrm{s} 2 \mathrm{~s}}
$$

Let $p$ be the pitch (distance from the center of one detector to the next) of a detector array and $L$ be the VIIRS effective focal length, and $\alpha$ be the angular interval. We have

$$
\alpha=p / L
$$

For an $n$ detectors of a band detector array, the band's ground projected field of regard (FOR), the along-track extent of the nadir ground projection of all detectors for a single sample, may be expressed as

$$
\mathrm{FOR}=n \alpha h
$$

where $h$ is the satellite altitude (and the angle $\alpha$ is assumed to be small).

The VIIRS nominal design has an angular interval $\alpha$ of $455.5 \mu$ rad in the track direction of I-band detectors ${ }^{[7]}$ and scan period $T$ of 1.786 seconds $^{[8]}$. Then the $\mathrm{V}_{\mathrm{s} 2 \mathrm{~s}}$ and $h$ in Fig. 7 can be converted to nominal (nom) FOR and $\mathrm{D}_{\mathrm{s} 2 \mathrm{~s}}$ using Eqs. (2) and (4). Fig. 8 shows the variations of $\mathrm{D}_{\mathrm{s} 2 \mathrm{~s}}$ and FOR with nominal ("nom" in the legend and that is with respect to instrument build) values shown in solid curves over one orbit. When $\mathrm{D}_{\mathrm{s} 2 \mathrm{~s}}$ and FOR are the same size, there is no gap (underlap) or overlap between adjacent VIIRS scans at nadir. When the FOR is larger there is a scan-to-scan overlap and when the FOR is smaller there is an underlap. Some underlap appears over the equator region (Fig. 8), with the maximum gap around 15 degrees latitude north. Fig. 8 also shows the sensitivity of the overlap and underlap on the orbit altitude and the VIIRS design (or as-built) parameters such as scan period and angular interval. If the VIIRS is built with FOR $1 \%$ larger and scan period $1 \%$ shorter (thus $\mathrm{D}_{\mathrm{s} 2 \mathrm{~s}} 1 \%$ smaller), there will be overlap everywhere. On the other hand, if the VIIRS is built with FOR $1 \%$ smaller and scan period $1 \%$ longer (thus $\mathrm{D}_{\mathrm{s} 2 \mathrm{~s}} 1 \%$ larger), then there will be underlap everywhere except over the south polar region below around 50 degrees south.

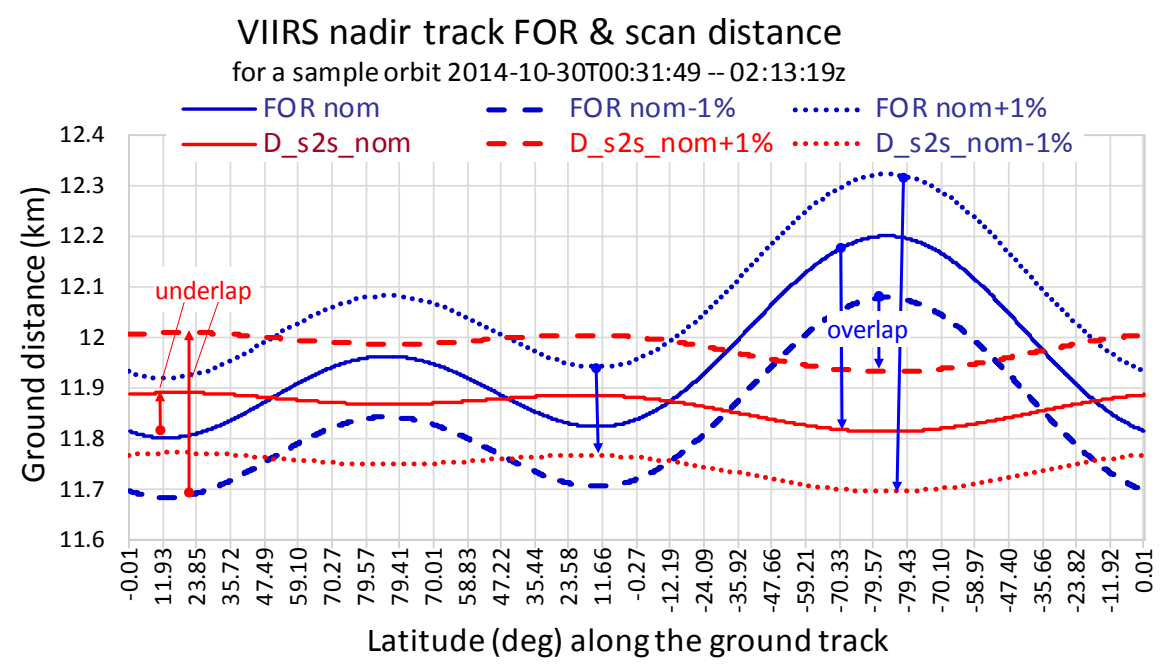

Fig. 8. Nominal (nom) scan-to-scan overlap and underlap at nadir within a sample orbit. Also shown is the sensitivity to variations of $+/-1 \%$ in the instrument parameters such as scan period and angular interval.

From Figs. 7 and 8, we can see that the maximum possible underlap occurs at the maximum speed, which corresponds to the location of minimum altitude in our case, at around 15 degrees latitude north in this sample orbit. Fig. 9 shows the mean and maximum $\mathrm{V}_{\mathrm{s} 2 \mathrm{~s}}$ for SNPP. The (long term) temporal variations in both the orbital mean and maximum values are small. The maximum speed in the VIIRS track direction may be taken as $6656 \mathrm{~m} / \mathrm{s}$. This is an empirical value from nearly 5 years of SNPP operations. The orbit parameters for the follow-on four JPSS satellites are expected to be the same as the SNPP. 


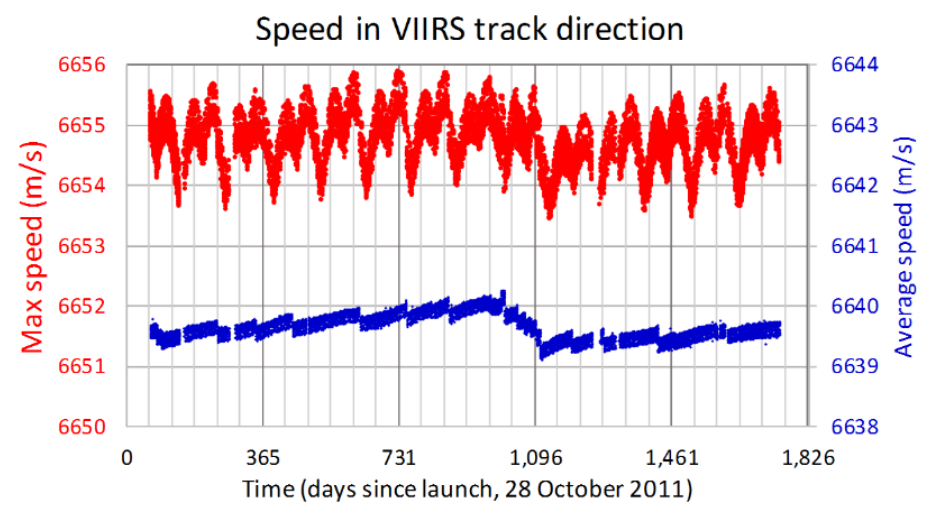

Fig. 9. $\mathrm{V}_{\mathrm{s} 2 \mathrm{~s}}$ trends for SNPP.

Taking the SNPP experience of the maximum $\mathrm{V}_{\mathrm{s} 2 \mathrm{~s}}$ of $6656 \mathrm{~m} / \mathrm{s}$ (Fig. 9 ) and minimum altitude of $828 \mathrm{~km}$ (Fig. 1(c)), we can also show the scan-to-scan overlap and underlap as a function of off-nadir scan angle. Fig. 10 shows the FOR in the black curve and the maximum $\mathrm{D}_{\mathrm{s} 2 \mathrm{~s}}$ at the maximum $\mathrm{V}_{\mathrm{s} 2 \mathrm{~s}}$ in the black dash line with nominal VIIRS parameters. We see that there is a small underlap with nominal VIIRS parameters with maximum underlap of $\sim 90 \mathrm{~m}$ extending to both sides off-nadir and closing at about $100 \mathrm{~km}$. Fig. 10 also shows the sensitivity of overlap and underlap on the VIIRS design (or as-built) parameters such as scan period and angular interval. If the VIIRS is built with FOR $1 \%$ larger and scan period $1 \%$ shorter (thus $\mathrm{D}_{\mathrm{s} 2 \mathrm{~s}} 1 \%$ smaller), there will be an overlap of $140 \mathrm{~m}$ at nadir and more off-nadir. On the other hand, if VIIRS is built with an FOR 1\% smaller but scan period (thus $\mathrm{D}_{\mathrm{s} 2 \mathrm{~s}}$ ) nominal, the gap at nadir would be $\sim 0.21 \mathrm{~km}$, or more than $50 \%$ of an I-band sample in the track direction. This gap would then close at $\sim 150 \mathrm{~km}$ from nadir at the ascending node in our sample orbit (Fig. 8). Fig. 8 also shows that the gap at nadir would not close in the northern hemisphere (minimum nadir gap would be $\sim 20 \mathrm{~m}$ ), but would close at $\sim 35^{\circ} \mathrm{S}$ in the southern hemisphere.

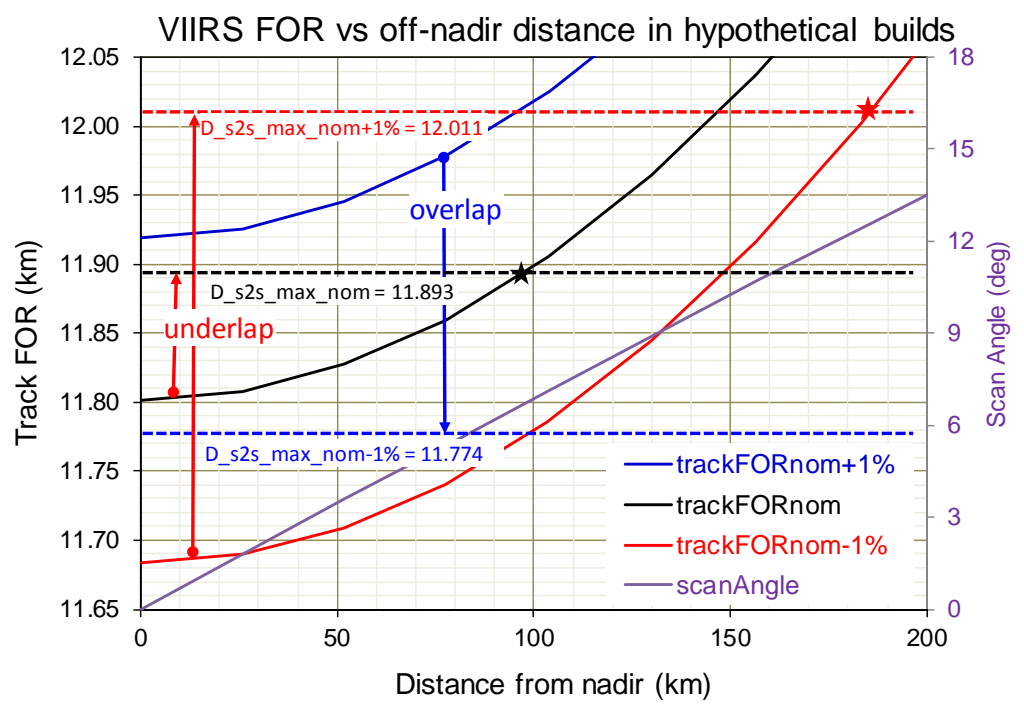

Fig. 10. Sensitivity of scan-to-scan overlap and underlap to VIIRS design parameters as a function of distance from nadir at the assumed minimum satellite altitude of $828 \mathrm{~km}$ and maximum $\mathrm{V}_{\mathrm{s} 2 \mathrm{~s}}$ of $6656 \mathrm{~m} / \mathrm{s}$.

Combining Figs. 8 and 10, we can study the extent of scan-to-scan underlap in the off-nadir direction in adjacent scans and in the global coverage along the SSP ground tracks. Using the nominal design parameters a scan-to-scan gap occur and the shape would look like a long "cat" eye extending from nadir to both off-nadir sides in the scan direction. Along the ground tracks, that cat eye would start opening from the south hemisphere approaching the equator in the ascending orbit and reach its maximum at about $15^{\circ} \mathrm{N}$. The size would gradually decease and close at $48^{\circ} \mathrm{N}$. In the descending orbit, the eye would start opening again at $45^{\circ} \mathrm{N}$, reach its maximum at $15^{\circ} \mathrm{N}$, then close at $12^{\circ} \mathrm{S}$. 


\section{DISCUSSIONS AND CONCLUDING REMARKS}

This paper reports the trending results of the SNPP orbit parameters. The orbit parameters such as LTAN, inclination angle and orbit mean parameters (position from the earth center, altitude and speed) show that the orbit is "frozen" in the sense that these parameters vary by only a small amount over the SNPP mission and are well controlled by station-keeping operations (DMUs and IAMs). JPSS-1 satellite with the second VIIRS build as one of the payloads is planned to be launched in 2017 in the same orbit as the SNPP with a definitive phasing.

Different from the small temporal variabilities of the SNPP orbit mean parameters, the within-orbit variabilities of these parameters are significant. A few observed features of these variabilities are:

1) The orbit in ECI is an ellipse in which the perigee (relative to the earth center) is near the north pole, the apogee is near the south pole, the center is about $8 \mathrm{~km}$ south of the earth center, and the longer axis ( $6 \mathrm{~km}$ longer) is the line that goes through the center and extends to the ascending and descending nodes;

2) The spacecraft speed in ECI has its peak near the ascending or descending node (not at perigee), and its trough at apogee;

3) The altitude has its minimum near the ascending or descending node (not at its perigee) and its peak is at apogee;

4) The ground speed has its peak near the ascending and descending nodes.

The SNPP orbit does not follow the laws of a Keplerian elliptic orbit in a 2-body dynamics. This is due to the Earth's oblateness and other perturbations.

Since the orbit is not truly polar, earth rotation contributes speed to the SSP speed and the variations in $V_{\text {s2s }}$ and the corresponding nadir scan-to-scan center distance, $\mathrm{D}_{\mathrm{s} 2 \mathrm{~s}}$. $\mathrm{V}_{\mathrm{s} 2 \mathrm{~s}}$ is the same as the satellite SSP speed only at the northernmost and southernmost parts of the orbit, and is $0.24 \%$ smaller than the maximum satellite SSP speed near the equator. The contribution of the earth rotation to the $\mathrm{V}_{\mathrm{s} 2 \mathrm{~s}}$ is $1.1 \%$ of the $\mathrm{V}_{\mathrm{ECI}}$.

The scan-to-scan underlap and overlap is sensitive to variations in as-built instrument parameters such as scan period and focal length. Variations in instrument parameters as small as $1 \%$ may cause near-nadir gaps larger than $50 \%$ of a sample in a VIIRS-like instrument in a retrograde near-polar orbit.

Based on the this analysis, we recommend that when planning for future missions, engineers should include the maximum ground speed that takes full account of earth rotation in the designs of VIIRS-like instruments carried on retrograde nearpolar orbiting satellites to ensure that there is no gap between scans and thereby ensuring contiguous coverage.

\section{ACKNOWLEDGEMENTS}

The authors are thankful to Bin Tan of SSAI/GSFC and Fred Patt of SAIC/GSFC for helpful discussions in the ground track speeds for VIIRS and SNPP satellite and their frames of references.

The authors acknowledge the sponsorship of this work by the JPSS Project Science Office and NOAA Center for Satellite Applications and Research (STAR). The manuscript contents are solely the opinions of the authors and do not constitute a statement of policy, decision, or position on behalf of SSAI, NASA or the U.S. government.

\section{REFERENCES}

[1] Wolfe, R.E., G. Lin, M.Nishihama, K.P. Tewari, E. Montano (2012), "NPP VIIRS Early On-Orbit Geometric Performance”, Earth Observing Systems XVII, edited by J. J. Butler, X. Xiong, X. Gu, Proc. of SPIE Vol. 8510, 851013, doi: 10.1117/12.929925, Oct. 2012.

[2] Wolfe, R.E., G. Lin, M. Nishihama, K. P. Tewari, J. C. Tilton, A. R. Isaacman (2013), "Suomi NPP VIIRS prelaunch and on-orbit geometric calibration and characterization”, Journal of Geophysical Research - Atmospheres, VOL. 118, 11,508-11,521, doi:10.1002/jgrd.50873, 2013.

[3] Cao, C., F. DeLuccia, X. Xiong, R. Wolfe, F. Weng (2014), "Early On-orbit Performance of the Visible Infrared Imaging Radiometer Suite (VIIRS) onboard the Suomi National Polar-orbiting Partnership (S-NPP) Satellite,” IEEE Trans. Geosci. Remote Sens., vol. 52, no. 2, pp. 1142-1156, DOI:10,1109/TGRS.2013.2247768, Feb. 2014, 
[4] Cao, C., X. Xiong, R. Wolfe, F. Deluccia, Q. Liu, S. Blonski, G. Lin, M., Nishihama, D. Pogorzala, and H. Oudrari, D. Hillger (2013), "Visible Infrared Imaging Radiometer Suite (VIIRS) Sensor Data Record (SDR) User's Guide," U.S. Dept. Commerce, NOAA, Silver Spring, MD, USA, Tech. Rep. NESDIS 142, Ver. 1.2, September 2013.

[5] Howard D. Curtis (2011), Orbital Mechanics: for Engineering Students. Elsevier.

[6] Karl D. Bilimoria and Rogier A. Krieger (2011), "Slot Architecture for Separating Satellites in Sun-Synchronous Orbits”, AIAA SPACE 2011 Conference \& Exposition.

[7] Lin, G., R. E. Wolfe, M. Nishihama (2011), "NPP VIIRS Geometric Performance Status,” Earth Observing Systems XVI, edited by James J. Butler, Xiaoxiong Xiong, Xingfa Gu, Proc. of SPIE, Vol. 8153, pp. 81531V-81531V-14, doi: 10.1117/12.894652, Sept. 2011.

[8] Lin, G., R. E. Wolfe (2016), “JPSS-1 VIIRS at-launch geometric performance”, this issue of the proceedings. 